\title{
The genome sequence of the heath fritillary, Melitaea athalia
}

\section{(Rottemburg, 1775) [version 1; peer review: 2 approved]}

\author{
Alex Hayward1', Roger Vila (D2), Dominik R. Laetsch (D)3, Konrad Lohse3, \\ Tobias Baril(D1, Darwin Tree of Life Barcoding collective, \\ Wellcome Sanger Institute Tree of Life programme, \\ Wellcome Sanger Institute Scientific Operations: DNA Pipelines collective, \\ Tree of Life Core Informatics collective, Darwin Tree of Life Consortium

\footnotetext{
${ }^{1}$ University of Exeter, Penryn, UK

${ }^{2}$ Institut de Biologia Evolutiva (CSIC - Universitat Pompeu Fabra), Barcelona, Spain

${ }^{3}$ Institute of Evolutionary Biology, University of Edinburgh, Edinburgh, UK
}

\begin{tabular}{l} 
V1 First published: 10 Nov 2021, 6:304 \\
https://doi.org/10.12688/wellcomeopenres.17280.1 \\
Latest published: 10 Nov 2021, 6:304 \\
https://doi.org/10.12688/wellcomeopenres.17280.1 \\
\hline
\end{tabular}

\section{Abstract}

We present a genome assembly from an individual female Melitaea athalia (also known as Mellicta athalia; the heath fritillary; Arthropoda; Insecta; Lepidoptera; Nymphalidae). The genome sequence is 610 megabases in span. In total, $99.98 \%$ of the assembly is scaffolded into 32 chromosomal pseudomolecules, with the $\mathrm{W}$ and $\mathrm{Z}$ sex chromosome assembled. Gene annotation of this assembly on Ensembl has identified 12,824 protein coding genes.

Keywords

Melitaea athalia, Mellicta athalia, heath fritillary, genome sequence, chromosomal

This article is included in the Tree of Life gateway.

\section{Open Peer Review \\ Approval Status \\ 1 2 \\ version 1 \\ 10 Nov 2021

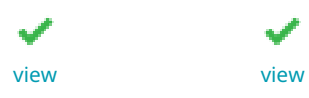 \\ 1. Brian A. Counterman (D), Auburn University, Auburn, USA \\ 2. Hikmet Budak Montana BioAgriculture Inc., Missoula, USA}

Any reports and responses or comments on the article can be found at the end of the article. 
Corresponding author: Darwin Tree of Life Consortium (mark.blaxter@sanger.ac.uk)

Author roles: Hayward A: Investigation, Resources; Vila R: Investigation, Resources, Writing - Review \& Editing; Laetsch DR: Investigation, Resources; Lohse K: Investigation, Resources, Writing - Review \& Editing; Baril T: Writing - Original Draft Preparation, Writing - Review \& Editing;

Competing interests: No competing interests were disclosed.

Grant information: This work was supported by Wellcome through core funding to the Wellcome Sanger Institute (206194) and the Darwin Tree of Life Discretionary Award (218328). KL is supported by a NERC fellowship (NE/L011522/1) and an ERC grant (ModelGenom Land 757648) which also supported fieldwork in Romania. AH is supported by a Biotechnology and Biological Sciences Research Council (BBSRC) David Phillips Fellowship (BB/N020146/1). TB is supported by a studentship from the Biotechnology and Biological Sciences Research Council-funded South West Biosciences Doctoral Training Partnership (BB/M009122/1).

The funders had no role in study design, data collection and analysis, decision to publish, or preparation of the manuscript.

Copyright: ( 2021 Hayward A et al. This is an open access article distributed under the terms of the Creative Commons Attribution License, which permits unrestricted use, distribution, and reproduction in any medium, provided the original work is properly cited.

How to cite this article: Hayward A, Vila R, Laetsch DR et al. The genome sequence of the heath fritillary, Melitaea athalia (Rottemburg, 1775) [version 1; peer review: 2 approved] Wellcome Open Research 2021, 6:304

https://doi.org/10.12688/wellcomeopenres.17280.1

First published: 10 Nov 2021, 6:304 https://doi.org/10.12688/wellcomeopenres.17280.1 


\section{Species taxonomy}

Eukaryota; Metazoa; Ecdysozoa; Arthropoda; Hexapoda; Insecta; Pterygota; Neoptera; Endopterygota; Lepidoptera; Glossata; Ditrysia; Papilionoidea; Nymphalidae; Nymphalinae; Melitaea athalia (also known as Mellicta athalia) (Rottemburg, 1775) (NCBI:txid113330).

\section{Introduction}

The heath fritillary, Melitaea athalia (also known as Mellicta athalia), is a medium-small sized butterfly found throughout the Palaearctic from western Europe to Japan. Historically, the species has been linked with the traditional practice of woodland coppicing, earning it the nickname of 'Woodman's Follower'. M. athalia is one of the UK's rarest butterflies and was on the brink of extinction during the 1970s, but conservation efforts have since helped to save the species (Warren, 1987). In the UK M. athalia is restricted to grasslands in Cornwall and Devon, heathland in Exmoor, and coppiced woodland in Kent and Essex (Tomlinson \& Still, 2002) and is a species of principal importance under the Natural Environment and Rural Communities Act 2006. However, it is listed as Least Concern in the IUCN Red List (Europe) (van Swaay et al., 2010). Up to eight forms and subspecies are recognized in Europe (Tolman \& Lewington, 1997). The taxon celadussa Fruhstorfer, 1910, originally described as a subspecies of athalia from southwestern Europe, is now recognized by many authors as a distinct parapatric species, with a contact zone extending from France to Austria where hybrids are found (Wiemers et al., 2018). Univoltine Fennoscandian and southern European alpine subspecies fly in single broods (June-July), whilst subalpine subspecies are bivoltine and fly during May-June and late July-August (Tolman \& Lewington, 1997). Females of $M$. athalia lay eggs in batches on the underside of leaves of a wide range of herbaceus food plants, with caterpillars feeding, aestivating, and hibernating together in silk nests (Wahlberg, 2000). The standard haploid karyotype of $M$. athalia consists of 30 autosomes and one sex chromsome (Bátori et al., 2012), and the female is heterogametic (WZ).

\section{Genome sequence report}

The genome was sequenced from a single female $M$. athalia collected from Lupşa, Transylvania, Romania (latitude 46.416, longitude 23.192) (Figure 1). A total of 30-fold coverage in Pacific Biosciences single-molecule long reads (N50 $16 \mathrm{~kb}$ ) and 64-fold coverage in 10X Genomics read clouds were generated. Primary assembly contigs were scaffolded with chromosome conformation Hi-C data. Manual assembly curation corrected $82 \mathrm{missing} / \mathrm{misjoins}$ and removed 19 haplotypic duplications, reducing the assembly size by $1.94 \%$ and scaffold number by $45.12 \%$, and increasing the scaffold N50 by $7.20 \%$.

The final assembly has a total length of $610 \mathrm{Mb}$ in 46 sequence scaffolds with a scaffold $\mathrm{N} 50$ of $20 \mathrm{Mb}$ (Table 1). Of the assembly sequence, $99.98 \%$ was assigned to 32 chromosomal-level scaffolds, representing 30 autosomes (numbered by sequence length), and the $\mathrm{W}$ and $\mathrm{Z}$ sex chromosome (Figure 2-Figure 5; Table 2). The assembly has a BUSCO (Simão et al., 2015)
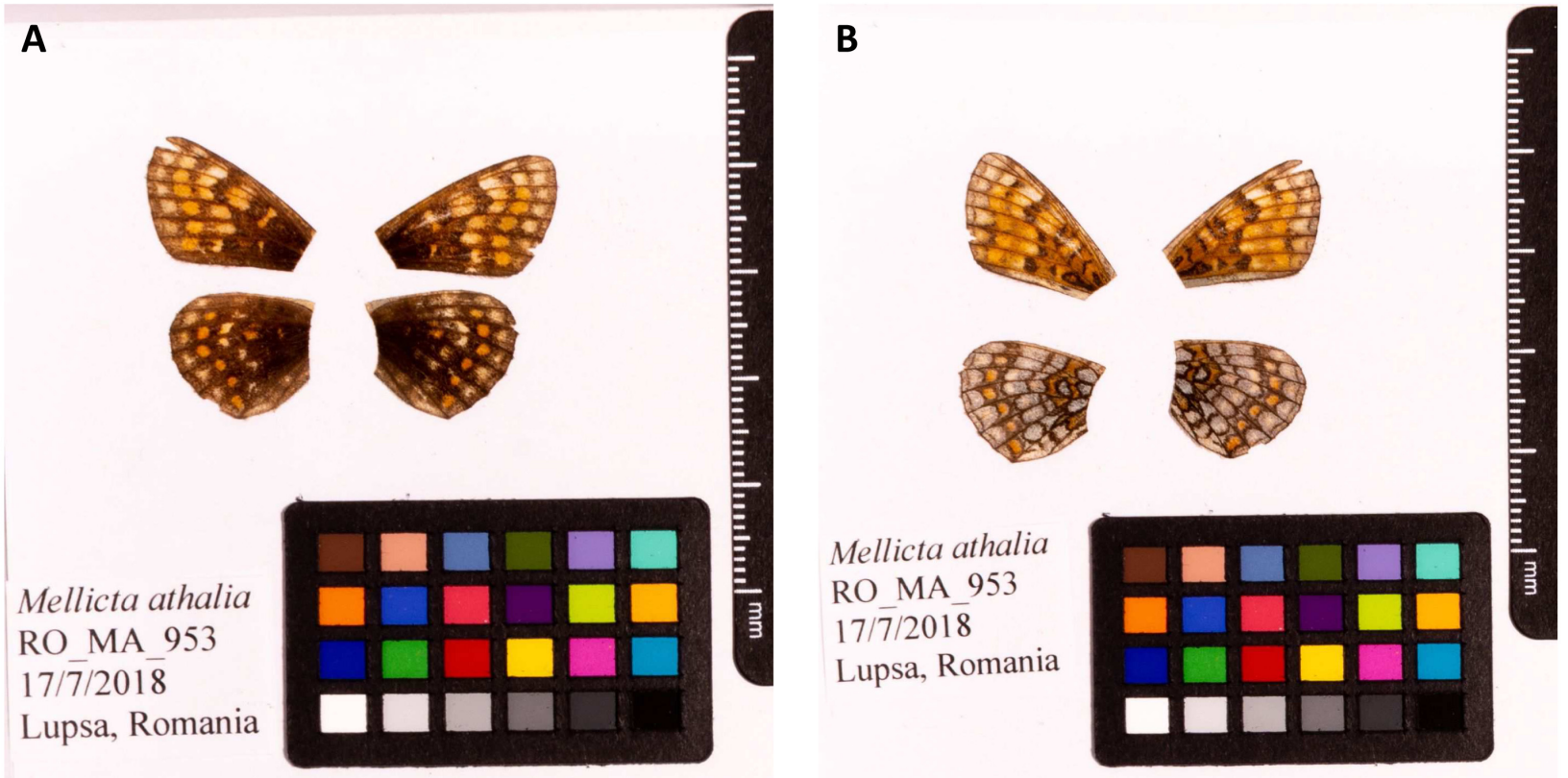

Figure 1. Fore and hind wings of Melitaea athalia specimen from which the genome was sequenced. (A) Dorsal surface view of wings from specimen RO_MA_953 (ilMelAtha1.1) from Lupşa, Transylvania, Romania, used to generate Pacific Biosciences and 10X genomics data. (B) Ventral surface view of wings from specimen RO_MA_953 (ilMelAtha1.1) from Lupşa, Transylvania, Romania, used to generate Pacific Biosciences and 10X genomics data. 
Table 1. Genome data for Melitaea athalia, ilMelAtha1.1.

\begin{tabular}{|c|c|}
\hline \multicolumn{2}{|l|}{ Project accession data } \\
\hline Assembly identifier & ilMelAtha1.1 \\
\hline Species & Melitaea athalia (also known as Mellicta athalia) \\
\hline Specimen & ilMelAtha1, RO_MA_953 \\
\hline NCBI taxonomy ID & NCBI:txid113330 \\
\hline BioProject & PRJEB42954 \\
\hline BioSample ID & SAMEA7523312 \\
\hline Isolate information & Female, whole organism \\
\hline \multicolumn{2}{|l|}{ Raw data accessions } \\
\hline PacificBiosciences SEQUEL II & ERR6576319 \\
\hline 10X Genomics Illumina & ERR6054423-ERR6054426 \\
\hline Hi-C Illumina & ERR6054427 \\
\hline \multicolumn{2}{|l|}{ Genome assembly } \\
\hline Assembly accession & GCA_905163435.1 \\
\hline Accession of alternate haplotype & GCA_905163405.1 \\
\hline Span (Mb) & 576 \\
\hline Number of contigs & 70 \\
\hline Contig N50 length (Mb) & 18 \\
\hline Number of scaffolds & 43 \\
\hline Scaffold N50 length (Mb) & 19 \\
\hline Longest scaffold (Mb) & 23 \\
\hline BUSCO* genome score & C:98.6\%[S:97.9\%,D:0.7\%],F:0.4\%,M:1.0\%,n:5286 \\
\hline \multicolumn{2}{|l|}{ Gene annotation } \\
\hline Number of protein coding genes & 12,824 \\
\hline Average coding sequence length (bp) & 1,492 \\
\hline Average number of exons per transcript & 8 \\
\hline Average exon size (bp) & 264 \\
\hline Average intron size (bp) & 2,892 \\
\hline
\end{tabular}

completeness of $98.6 \%$ (single $97.9 \%$, duplicated $0.7 \%$, fragmented $0.4 \%$, missing $1.0 \%$ ) using the lepidoptera_odb10 reference set. While not fully phased, the assembly deposited is of one haplotype. Contigs corresponding to the second haplotype have also been deposited.

\section{Gene annotation}

The Ensembl gene annotation system (Aken et al., 2016) was used to generate annotation for the Melitaea athalia assembly
(GCA_905220545.1, see https://rapid.ensembl.org/Mellicta_ athalia_GCA_905220545.1/; Table 1). The annotation was created primarily through alignment of transcriptomic data to the genome, with gap filling via protein-to-genome alignments of a select set of proteins from UniProt (UniProt Consortium, 2019)) and OrthoDB (Kriventseva et al., 2008). Prediction tools, CPC2 (Kang et al., 2017) and RNAsamba (Camargo et al., 2020), were used to aid determination of protein coding genes. 


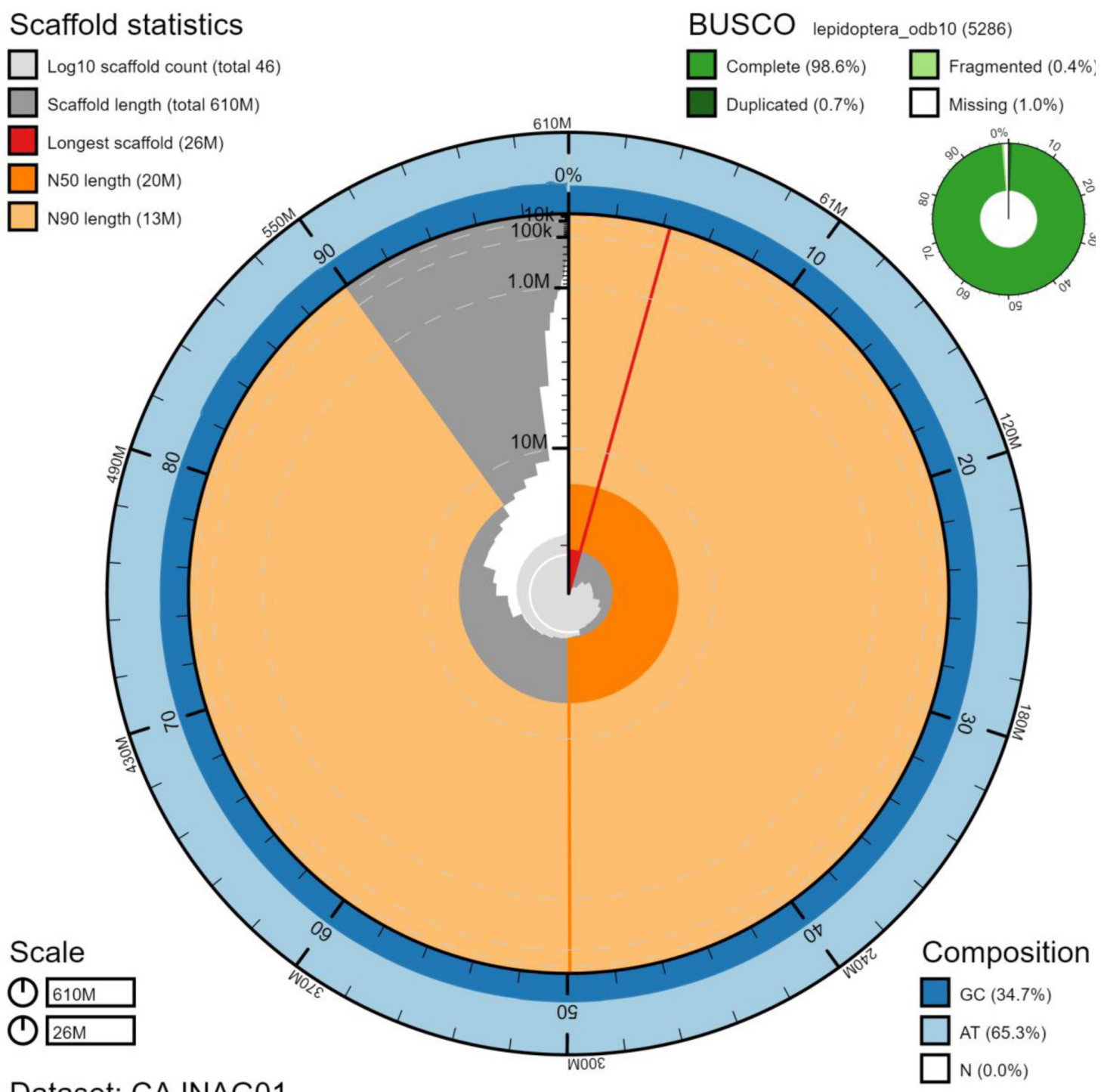

\section{Dataset: CAJNAG01}

Figure 2. Genome assembly of Mellitaea athalia, ilMelAtha1.1: metrics. The BlobToolKit Snailplot shows N50 metrics and BUSCO gene completeness. The main plot is divided into 1,000 size-ordered bins around the circumference with each bin representing $0.1 \%$ of the $609,564,789 \mathrm{bp}$ assembly. The distribution of scaffold lengths is shown in dark grey with the plot radius scaled to the longest chromosome present in the assembly (26,233,870 bp, shown in red). Orange and pale-orange arcs show the N50 and N90 scaffold lengths $(20,295,254$ and $13,271,753$ bp), respectively. The pale grey spiral shows the cumulative scaffold count on a log scale with white scale lines showing successive orders of magnitude. The blue and pale-blue area around the outside of the plot shows the distribution of GC, AT and $\mathrm{N}$ percentages in the same bins as the inner plot. A summary of complete, fragmented, duplicated and missing BUSCO genes in the lepidoptera_odb10 set is shown in the top right. An interactive version of this figure is available at https://blobtoolkit.genomehubs.org/view/ ilMelAtha1.1/dataset/CAJNAG01/snail.

\section{Methods}

Sample acquisition, nucleic acid extraction and sequencing

A single female $M$. athalia was collected from Lupşa, Transylvania, Romania (latitude 46.416, longitude 23.192) by Alex Hayward (University of Exeter), Roger Vila (Universitat
Pompeu Fabra), Dominik Laetsch and Konrad Lohse (both University of Edinburgh), using a net. The specimen was identified by Roger Vila and was snap-frozen in liquid nitrogen.

DNA was extracted from the whole organism of ilMelAtha1 using the Qiagen MagAttract HMW DNA kit, according to 


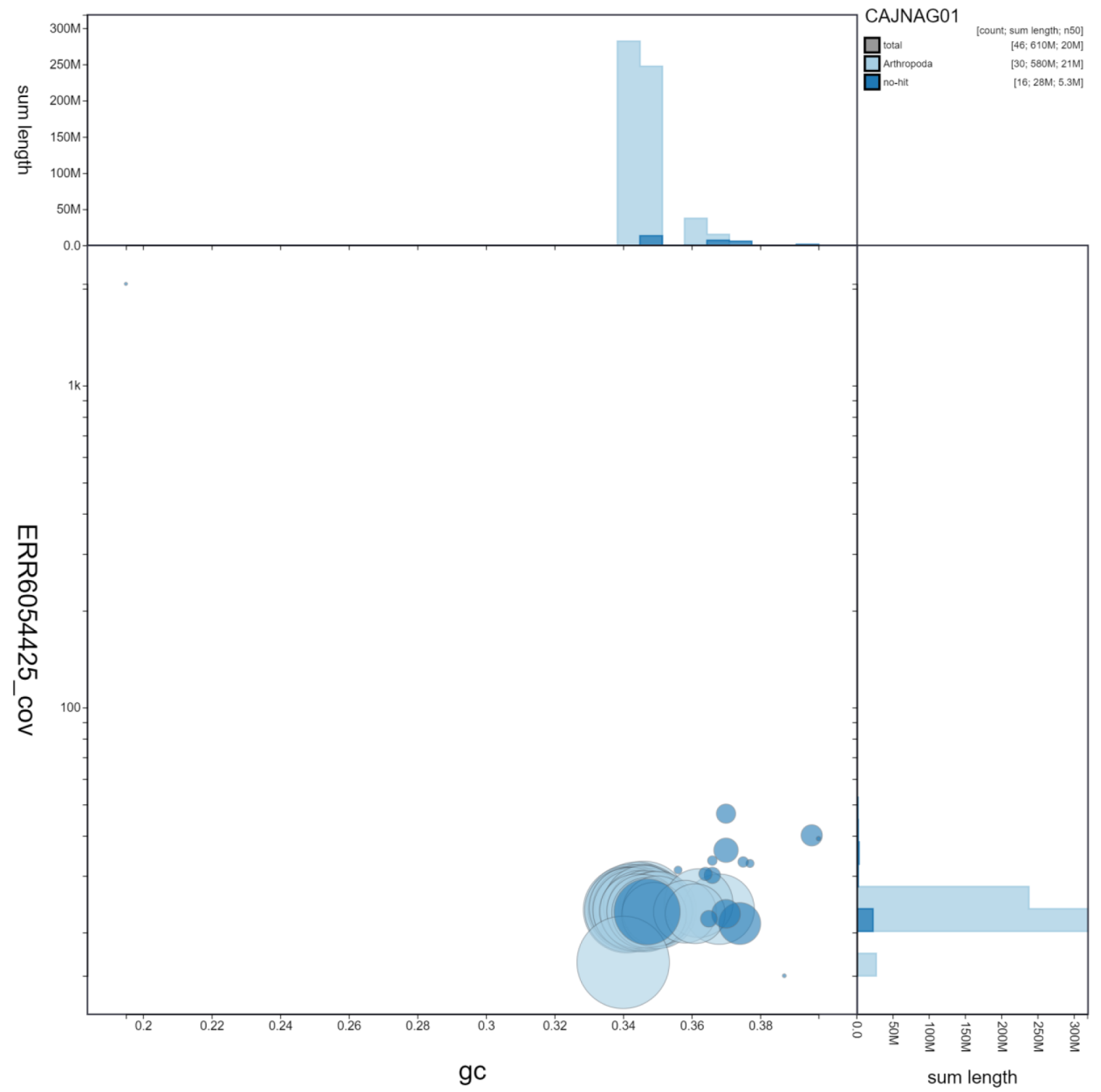

Figure 3. Genome assembly of Mellitaea athalia , ilMelAtha1.1: GC coverage. BlobToolKit GC-coverage plot. Scaffolds are coloured by phylum. Circles are sized in proportion to scaffold length. Histograms show the distribution of scaffold length sum along each axis. An interactive version of this figure is available at https://blobtoolkit.genomehubs.org/view/ilMelAtha1.1/dataset/CAJNAG01/blob.

the manufacturer's instructions. Pacific Biosciences HiFi circular consensus and 10X Genomics read cloud sequencing libraries were then constructed according to the manufacturers' instructions. Sequencing was performed by the Scientific Operations core at the Wellcome Sanger Institute on Pacific Biosciences SEQUEL II and Illumina HiSeq X instruments. Hi-C data were generated using the Arima v1.0 kit and sequenced on HiSeq X.

\section{Genome assembly}

Assembly was carried out with HiCanu (Nurk et al., 2020). Haplotypic duplication was identified and removed with purge_dups (Guan et al., 2020). One round of polishing was performed by aligning $10 \mathrm{X}$ Genomics read data to the assembly with longranger align, calling variants with freebayes (Garrison \& Marth, 2012). The assembly was then scaffolded with Hi-C data (Rao et al., 2014) using SALSA2 (Ghurye et al., 2019). The assembly was checked for contamination and corrected using the gEVAL system (Chow et al., 2016) as described previously (Howe et al., 2021). Manual curation was performed using gEVAL, HiGlass (Kerpedjiev et al., 2018) and Pretext. The mitochondrial genome was assembled using MitoHiFi (Uliano-Silva et al., 2021). The genome was analysed and BUSCO scores generated within the BlobToolKit 


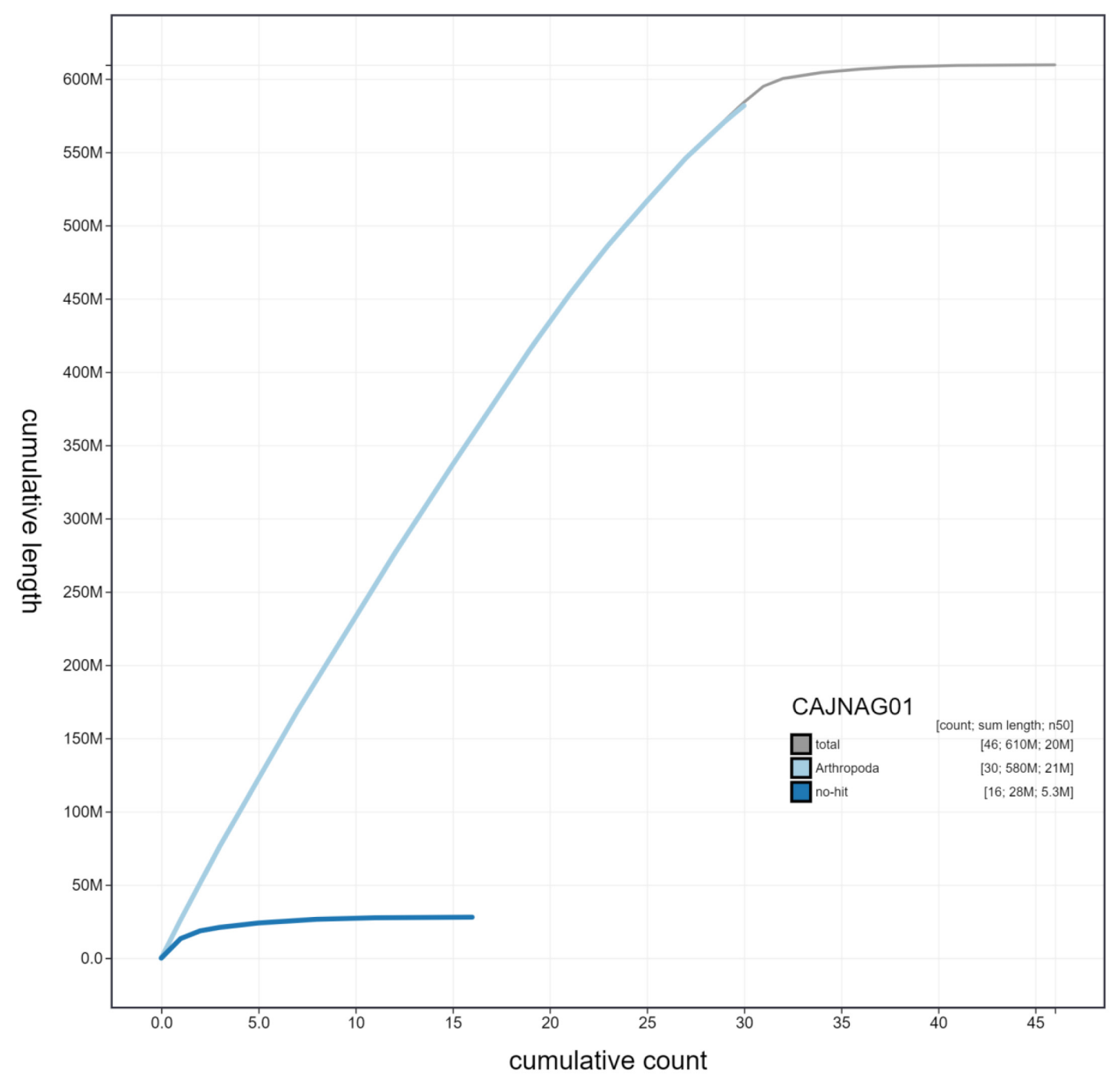

Figure 4. Genome assembly of Mellitaea athalia, ilMelAtha1.1: cumulative sequence. BlobToolKit cumulative sequence plot. The grey line shows cumulative length for all scaffolds. Coloured lines show cumulative lengths of scaffolds assigned to each phylum using the buscogenes taxrule. An interactive version of this figure is available at https://blobtoolkit.genomehubs.org/view/ilMelAtha1.1/dataset/ CAJNAG01/cumulative.

environment (Challis et al., 2020). Table 3 contains a list of all software tool versions used, where appropriate.

\section{Ethical/compliance issues}

The materials that have contributed to this genome note were supplied by a Tree of Life collaborator. The Wellcome Sanger Institute employs a process whereby due diligence is carried out proportionate to the nature of the materials themselves, and the circumstances under which they have been/are to be collected and provided for use. The purpose of this is to address and mitigate any potential legal and/or ethical implications of receipt and use of the materials as part of the research project, and to ensure that in doing so we align with best practice wherever possible.

The overarching areas of consideration are:

- Ethical review of provenance and sourcing of the material;

- Legality of collection, transfer and use (national and international). 


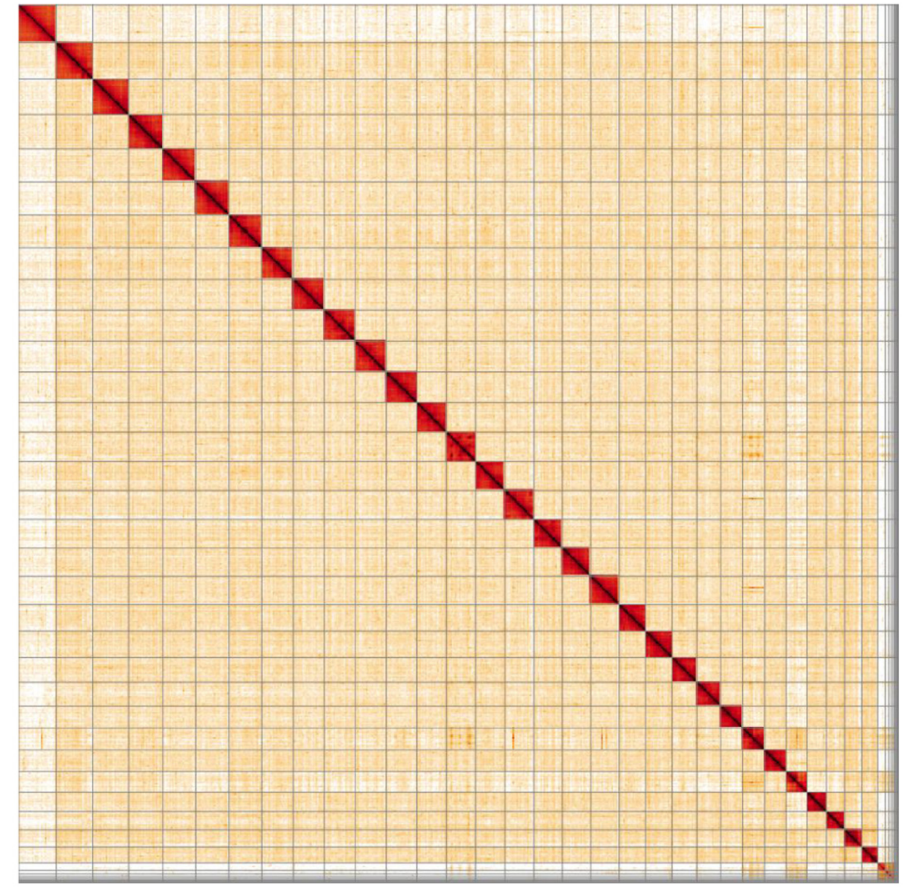

Figure 5. Genome assembly of Mellitaea athalia, ilMelAtha1.1: Hi-C contact map. Hi-C contact map of the ilMelAtha1.1 assembly, visualised in HiGlass. Chromosomes are arranged in size order from left to right and top to bottom.

\begin{tabular}{|c|c|c|c|}
\hline \multicolumn{3}{|c|}{$\begin{array}{l}\text { Table 2. Chromosomal pseudomolecules in the } \\
\text { genome assembly of Melitaea athalia, ilMelAtha1.1. }\end{array}$} \\
\hline INSDC accession & Chromosome & Size (Mb) & GC\% \\
\hline HG992177.1 & 1 & 25.13 & 34.6 \\
\hline HG992178.1 & 2 & 24.88 & 34.4 \\
\hline HG992179.1 & 3 & 23.65 & 34.6 \\
\hline HG992180.1 & 4 & 22.93 & 34.2 \\
\hline HG992181.1 & 5 & 22.90 & 34.1 \\
\hline HG992182.1 & 6 & 22.79 & 34.5 \\
\hline HG992183.1 & 7 & 21.87 & 34.4 \\
\hline HG992184.1 & 8 & 21.42 & 34.3 \\
\hline HG992185.1 & 9 & 21.39 & 34.2 \\
\hline HG992186.1 & 10 & 21.38 & 34.1 \\
\hline HG992187.1 & 11 & 21.23 & 34.4 \\
\hline HG992188.1 & 12 & 20.51 & 34.3 \\
\hline HG992189.1 & 13 & 20.30 & 34.8 \\
\hline HG992190.1 & 14 & 20.21 & 34.2 \\
\hline HG992191.1 & 15 & 19.99 & 34.3 \\
\hline HG992192.1 & 16 & 19.82 & 34.6 \\
\hline
\end{tabular}

\begin{tabular}{|c|c|c|c|}
\hline INSDC accession & Chromosome & Size (Mb) & GC\% \\
\hline HG992193.1 & 17 & 19.64 & 34.5 \\
\hline HG992194.1 & 18 & 19.55 & 34.7 \\
\hline HG992195.1 & 19 & 18.44 & 34.9 \\
\hline HG992196.1 & 20 & 18.37 & 35 \\
\hline HG992197.1 & 21 & 17.06 & 34.4 \\
\hline HG992198.1 & 22 & 16.62 & 34.6 \\
\hline HG992199.1 & 23 & 15.22 & 34.7 \\
\hline HG992200.1 & 24 & 15.15 & 36.8 \\
\hline HG992201.1 & 25 & 14.97 & 35 \\
\hline HG992202.1 & 26 & 14.40 & 36.2 \\
\hline HG992203.1 & 27 & 13.27 & 34.7 \\
\hline HG992204.1 & 28 & 12.76 & 34.9 \\
\hline HG992205.1 & 29 & 11.97 & 35.8 \\
\hline HG992206.1 & 30 & 10.90 & 36.1 \\
\hline HG992207.1 & W & 5.27 & 37.4 \\
\hline HG992176.1 & $Z$ & 26.23 & 34 \\
\hline HG992208.1 & MT & 0.02 & 19.7 \\
\hline- & Unplaced & 9.34 & 37.2 \\
\hline
\end{tabular}


Table 3. Software tools used.

\begin{tabular}{|l|l|l|}
\hline Software tool & Version & Source \\
\hline HiCanu & 2.1 & Nurk et al., 2020 \\
\hline purge_dups & 1.2 .3 & Guan et al., 2020 \\
\hline SALSA2 & 2.2 & Ghurye et al., 2019 \\
\hline Iongranger align & 2.2 .2 & $\begin{array}{l}\text { https://support.10xgenomics.com/genome-exome/software/pipelines/latest/advanced/ } \\
\text { other-pipelines }\end{array}$ \\
\hline freebayes & $1.3 .1-17$-gaa2ace8 & Garrison \& Marth, 2012 \\
\hline MitoHiFi & 1 & https://github.com/marcelauliano/MitoHiFi \\
\hline gEVAL & 2016 & Chow et al., 2016 \\
\hline HiGlass & 1.11 .6 & Kerpedjiev et al., 2018 \\
\hline PretextView & $0.1 . x$ & https://github.com/wtsi-hpag/PretextView \\
\hline BlobToolKit & 2.6 .2 & Challis et al., 2020 \\
\hline
\end{tabular}

Each transfer of samples is undertaken according to a Research Collaboration Agreement or Material Transfer Agreement entered into by the Tree of Life collaborator, Genome Research Limited (operating as the Wellcome Sanger Institute) and in some circumstances other Tree of Life collaborators.

\section{Data availability}

European Nucleotide Archive: Mellicta athalia (heath fritillary). Accession number PRJEB42954; https://identifiers.org/ena.embl/ PRJEB42954.

The genome sequence is released openly for reuse. The M. athalia genome sequencing initiative is part of the Darwin Tree of Life (DToL) project. All raw sequence data and the assembly have been deposited in INSDC databases. Raw data and assembly accession identifiers are reported in Table 1.

\section{Acknowledgements}

Members of the Darwin Tree of Life Barcoding collective are listed here: https://doi.org/10.5281/zenodo.4893704.

Members of the Wellcome Sanger Institute Tree of Life programme collective are listed here: https://doi.org/10.5281/ zenodo. 5377053 .

Members of Wellcome Sanger Institute Scientific Operations: DNA Pipelines collective are listed here: https://doi.org/10.5281/ zenodo.4790456.

Members of the Tree of Life Core Informatics collective are listed here: https://doi.org/10.5281/zenodo.5013542.

Members of the Darwin Tree of Life Consortium are listed here: https://doi.org/10.5281/zenodo.4783559.
Aken BL, Ayling S, Barrell D, et al: The Ensembl Gene Annotation System. Database (Oxford). 2016; 2016: baw093.

PubMed Abstract | Publisher Full Text | Free Full Text

Bátori E, Pecsenye K, Pál Tóth 」, et al:: Patterns of Genetic and Morphometric Differentiation in Melitaea (Mellicta) athalia (Lepidoptera: Nymphalidae).

Biol J Linn Soc. Linnean Society of London. 2012; 107(2): 398-413.

Publisher Full Text

Camargo AP, Sourkov V, Pereira GAG, et al:: RNAsamba: Neural NetworkBased Assessment of the Protein-Coding Potential of RNA Sequences. NAR Genom Bioinform. 2020; 2(1): Iqz024.

PubMed Abstract | Publisher Full Text | Free Full Text

Challis R, Richards E, Rajan J, et al.: BlobToolKit-Interactive Quality
Assessment of Genome Assemblies. G3 (Bethesda). 2020; 10(4): 1361-74. PubMed Abstract | Publisher Full Text | Free Full Text Chow W, Brugger K, Caccamo M, et al.: gEVAL - a Web-Based Browser for Evaluating Genome Assemblies. Bioinformatics. 2016; 32(16): 2508-10. PubMed Abstract | Publisher Full Text | Free Full Text Garrison E, Marth G: Haplotype-Based Variant Detection from Short-Read Sequencing. arXiv: 1207.3907. 2012. Reference Source

Ghurye J, Rhie A, Walenz BP, et al.: Integrating Hi-C Links with Assembly Graphs for Chromosome-Scale Assembly. PLoS Comput Biol. 2019; 15(8): e1007273.

PubMed Abstract | Publisher Full Text | Free Full Text 
Guan D, McCarthy SA, Wood J, et al.: Identifying and Removing Haplotypic Duplication in Primary Genome Assemblies. Bioinformatics. 2020; 36(9): 2896-98.

PubMed Abstract | Publisher Full Text | Free Full Text

Howe K, Chow W, Collins J, et al.: Significantly Improving the Quality of Genome Assemblies through Curation. GigaScience. 2021; 10(1): giaa153. PubMed Abstract | Publisher Full Text | Free Full Text

Kang YJ, Yang DC, Kong L, et al.: CPC2: A Fast and Accurate Coding Potential Calculator Based on Sequence Intrinsic Features. Nucleic Acids Res. 2017; 45(W1): W12-16.

PubMed Abstract | Publisher Full Text | Free Full Text

Kerpedjiev P, Abdennur N, Lekschas F, et al.: HiGlass: Web-Based Visual Exploration and Analysis of Genome Interaction Maps. Genome Biol. 2018; 19(1): 125.

PubMed Abstract | Publisher Full Text | Free Full Text

Kriventseva EV, Rahman N, Espinosa O, et al.: OrthoDB: The Hierarchical

Catalog of Eukaryotic Orthologs. Nucleic Acids Res. 2008; 36(Database issue): D271-75.

PubMed Abstract | Publisher Full Text | Free Full Text

Nurk S, Walenz BP, Rhie A, et al: HiCanu: Accurate Assembly of Segmental Duplications, Satellites, and Allelic Variants from High-Fidelity Long Reads. Genome Res. 2020; 30(9): 1291-1305.

PubMed Abstract | Publisher Full Text | Free Full Text

Rao SS, Huntley MH, Durand NC, et al.: A 3D Map of the Human Genome at Kilobase Resolution Reveals Principles of Chromatin Looping. Cell. 2014; 159(7): 1665-80.

PubMed Abstract | Publisher Full Text | Free Full Text

Simão FA, Waterhouse RM, Ioannidis P, et al.: BUSCO: Assessing Genome
Assembly and Annotation Completeness with Single-Copy Orthologs. Bioinformatics. 2015; 31(19): 3210-12.

PubMed Abstract | Publisher Full Text

Tolman T, Lewington R: Butterflies of Britain \& Europe. Collins Field Guide.

Harper Collins, London. 1997.

Reference Source

Tomlinson D, Still R: Britain's Butterflies. WILDGuides. 2002.

Reference Source

Uliano-Silva M, Gabriel Ferreira Nunes J, Krasheninnikova K, et al.: marcelauliano/MitoHiFi: mitohifi_v2.0. 2021.

Publisher Full Text

UniProt Consortium: UniProt: A Worldwide Hub of Protein Knowledge.

Nucleic Acids Res. 2019; 47(D1): D506-15.

PubMed Abstract | Publisher Full Text | Free Full Text

van Swaay C, Wynhoff I, Verovnik R, et al.: IUCN Red List of Threatened

Species: Melitaea Athalia. IUCN Red List of Threatened Species, 2010;

e.T174355A7056436.

Publisher Full Text

Wahlberg N: Comparative Descriptions of the Immature Stages and Ecology of Five Finnish Melitaeine Butterfly Species (Lepidoptera: Nymphalidae).

Entomologica Fennica. 2000; 11(3): 167-74.

Publisher Full Text

Warren MS: The Ecology and Conservation of the Heath Fritillary Butterfly, Mellicta athalia. III. Population Dynamics and the Effect of Habitat

Management. J Appl Ecol. 1987; 24(2): 499-513.

Publisher Full Text

Wiemers M, Balletto E, Dincă V, et al.: An updated checklist of the European Butterflies (Lepidoptera, Papilionoidea). Zookeys. 2018; (811): 9-45.

PubMed Abstract | Publisher Full Text | Free Full Text 


\section{Open Peer Review}

\section{Current Peer Review Status:}

\section{Version 1}

Reviewer Report 27 January 2022

https://doi.org/10.21956/wellcomeopenres.19104.r47141

(C) 2022 Budak H. This is an open access peer review report distributed under the terms of the Creative Commons Attribution License, which permits unrestricted use, distribution, and reproduction in any medium, provided the original work is properly cited.

\section{Hikmet Budak}

Department of Genomics and Genome Editing, Montana BioAgriculture Inc., Missoula, MT, USA

I believe that there is data which contributes to the community. Both the genome sequencing and assembly methods and applications looks good and presented well although it would be great to go into details a little more so that other scientists can use/compare/benefit form their experiences. I am unsure if Figure 4 is necessary to show cumulative seq. The authors were even able to assemble the $\mathrm{W}$ chromosome. This would definitely help sequencing and chromosome level assembly of wheat stem sawfly and orange wheat blossom midge, dangerous insects in the USA.

Is the rationale for creating the dataset(s) clearly described?

Yes

Are the protocols appropriate and is the work technically sound? Yes

Are sufficient details of methods and materials provided to allow replication by others? Yes

Are the datasets clearly presented in a useable and accessible format?

Yes

Competing Interests: No competing interests were disclosed.

Reviewer Expertise: Plant genomics and biology, Next generation sequencing and annotations, smallRNAs, microRNAs, LncRNAs.

I confirm that I have read this submission and believe that I have an appropriate level of expertise to confirm that it is of an acceptable scientific standard. 
Reviewer Report 25 November 2021

https://doi.org/10.21956/wellcomeopenres.19104.r47139

(C) 2021 Counterman B. This is an open access peer review report distributed under the terms of the Creative Commons Attribution License, which permits unrestricted use, distribution, and reproduction in any medium, provided the original work is properly cited.

\section{Brian A. Counterman}

Department of Biological Sciences, Auburn University, Auburn, AL, USA

This manuscript presents a concise report of the genome sequencing and assembly of Melitaea athalia, one of the rarest butterflies in Europe. The methods used are appropriate and appear to have been conducted appropriately. The result is a highly contiguous, chromosome-level assembly of the genome. It is noteworthy that the authors were able to assemble the $\mathrm{W}$ chromosome and release the unphased assemblies of both diploid copies. This genome is certain to be a valuable resource for comparative genomics of Lepidoptera and future conservation efforts of the species.

I found no major errors or concerns with the manuscript, and felt the tables and figures included sufficiently and succinctly presented relevant information to assess the methods used and quality of the genome assembly. I was unclear which version of BUSCO was used and if the "lepidoptera_odb10" database was the 2019 version. These details could be useful for future users of the data, and perhaps could be simply added to Table 3. Overall, I commend the authors for their clear and concise presentation of the work.

Is the rationale for creating the dataset(s) clearly described?

Yes

Are the protocols appropriate and is the work technically sound? Yes

Are sufficient details of methods and materials provided to allow replication by others? Yes

Are the datasets clearly presented in a useable and accessible format? Yes

Competing Interests: No competing interests were disclosed.

Reviewer Expertise: Lepidoptera, Evolution, Genomics

I confirm that I have read this submission and believe that I have an appropriate level of expertise to confirm that it is of an acceptable scientific standard. 